\title{
Shoulder biomechanics and the success of translational research
}

\author{
Andrea Giovanni Cutti · Edward K. Chadwick
}

Published online: 25 February 2014

(C) International Federation for Medical and Biological Engineering 2014

\begin{abstract}
In 2009, the International Shoulder Group (ISG) had the opportunity to propose to the readers of Medical and Biological Engineering and Computing a Special Issue on shoulder biomechanics. At that time, we pointed out that the field was evolving to include more applied research. After 4 years, we can confirm that impression: 10 out of 12 papers included in this second Special Issue deal with clinical related questions, through theoretical and experimental methodologies. This demonstrates that the translational research at the base of ISG foundation in 1989 is effective. We think that the papers of this issue will have an impact on clinics in general and on the treatment of work-related injuries and diseases in particular. Based on the statistics of the Italian Workers' Compensation Authority (INAIL), injuries at the shoulder are first in terms of average duration of "temporary total disability to work". Moreover, occupational diseases at the shoulder in the industrial and services sector represented $16 \%$ of all occupational diseases in 2012, i.e., $46 \%$ of those related to the upper limb. These data stress the need for specific interventions, with the contribution of both researchers and policy makers. Starting from the papers included here, we would encourage additional efforts on: (1) quantitative analysis of shoulder loading during tasks associated with musculoskeletal injuries, and ways to reduce that loading, (2) simple and effective tools to improve the diagnosis and outcome assessment of motion-related shoulder diseases, and (3) the
\end{abstract}

\footnotetext{
A. G. Cutti ( $\square)$

INAIL Prostheses Centre, Via Rabuina 14, 40054 Vigorso di Budrio, BO, Italy

e-mail: ag.cutti@inail.it

E. K. Chadwick

Institute for Science and Technology in Medicine,

Keele University, Staffordshire, UK
}

development of rehabilitation treatments focused on occupational tasks, taking advantage of state-of-the-art biofeedback technologies, and exploiting the power of biomechanical models for muscle force prediction.

\section{Introduction}

For the second time, the International Shoulder Group [17] has the opportunity to propose a Special Issue on shoulder biomechanics to the readers of Medical and Biological Engineering and Computing. The first time was after the conference in Bologna (Italy), with the Volume 47 Issue 5 in 2009 [8]. This time, it follows the 9th meeting in Aberystwyth (Wales), which set the scenery to a 3-day, single-session discussion on shoulder biomechanics by a multidisciplinary group of about 50 researchers from 12 nations.

The 2009 Special Issue collected a balanced mix of applied and fundamental, theoretical and experimental research, with 4 out of 12 papers in the applied/experimental corner (Fig. 1). This second Issue marks a trend: including the two review papers, 10 out of 12 papers are on the Applied Research side and 6 in the Applied and Experimental quadrant (Fig. 1). This demonstrates that the translational research at the base of ISG foundation in 1989 is effective.

\section{Fil rouge: the papers in perspective}

The papers in this issue also mark important technical changes in the field. The contributions of Braman [6] and de Witte [12], for instance, suggest that the diagnostic label "impingement syndrome" is misleading and uninformative. Is it time for its discontinuation, and definition of alternatives, in the interest of patient care? Specifically, 
Fig. 1 Classification of the papers included in this Special Issue, based on the content of each addressing fundamental of applied research questions, and using experimental or theoretical (mathematical modeling or simulation) methods. Black dots refer to the Special Issue in 2009 [8], while red dots refer to the present Special Issue (color figure online)

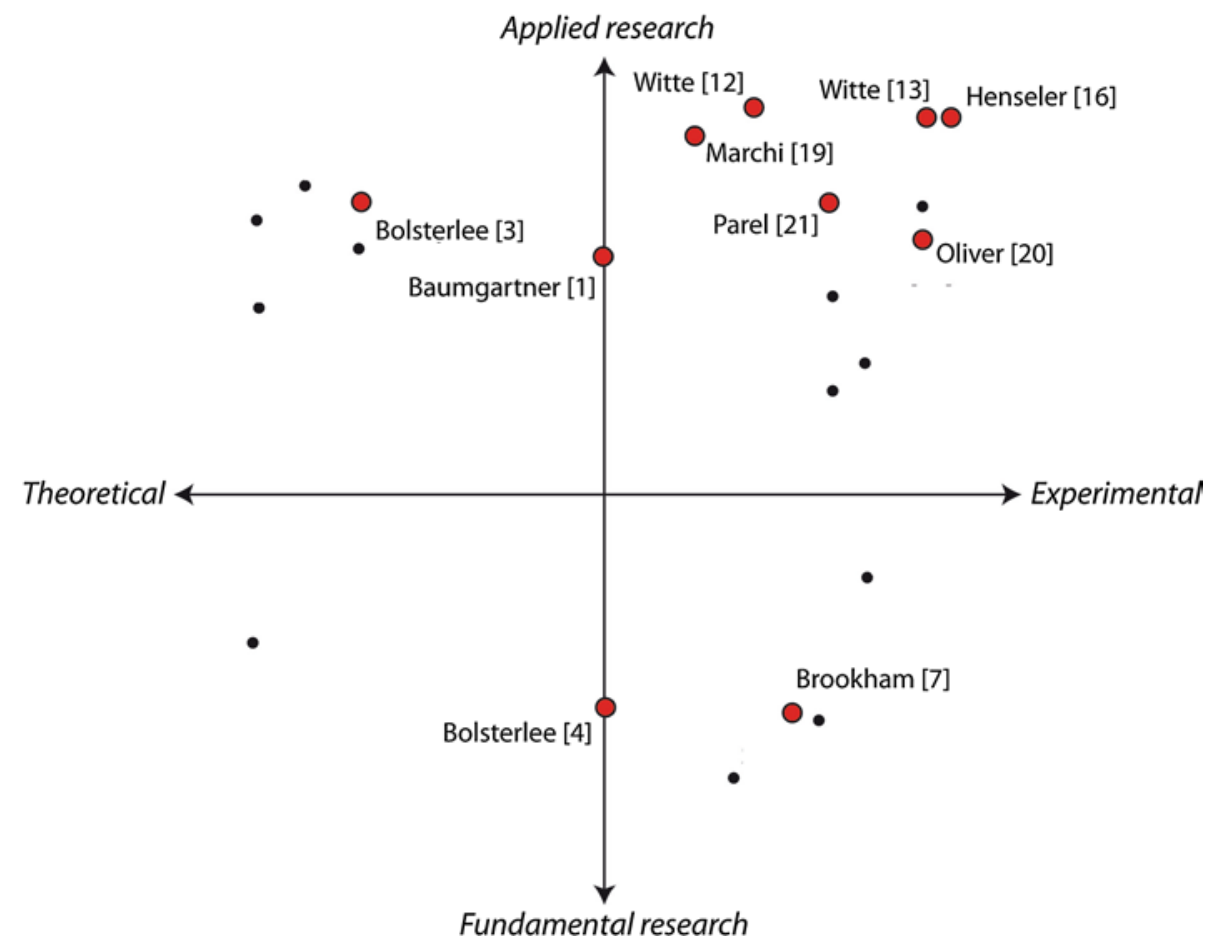

through a survey involving physical therapists and orthopedic surgeons from the USA and the Netherlands, de Witte et al. [12] concluded that clinicians from different professional backgrounds have different views on what subacromial impingement is in terms of etiology, diagnosis and treatment, and even within professional groups, variations are substantial. Interestingly, motion-related underlying mechanisms (dynamic factors) were associated with the diagnosis of subacromial impingement for physical therapists, while for orthopedic surgeons it seems to be more of an intrinsic, anatomical or structural problem (static factors). This increases the risk of misunderstandings and suboptimal patient care. Braman et al. [6] follow along this line and, based on a thorough examination of the literature, provide historical and practice-rated explanations for the divergence between professionals in the use of the label "shoulder impingement syndrome". Through a review of recent findings, they advocate the discontinuation of the term and the specific analysis of "mechanical impingement" and movement-related impairments. In particular, subgrouping patients based on physical examination findings might better direct patient treatment and ultimately improve outcomes. Moreover, the authors pointed out the importance of future longitudinal studies to better understand if identified movement abnormalities are causative, contributory or compensatory.

We would add that multicenter collaborations would give the possibility to run such longitudinal studies on a larger scale, including stratification criteria which might remain unexplored otherwise. However, a detailed analysis of agreement between methods and procedure applied in the different clinical centers should be carried out before initiation. This is an almost unexplored topic in motion analysis in general [2, 9, 14, 15], and for the upper extremity specifically. The paper by Parel et al. [21] gives a contribution on this regard, by assessing the agreement between two scapula-tracking systems (scapula tracker [18] and spine tracker [10]), based on two different motion analysis technologies, namely opto-eletronic and inertial and magnetic sensors [10, 11]. Filling a gap in current literature, the paper points out a clear set of conditions for agreement between systems for scapula tracking, based on state-ofthe-art parameters, i.e., within-protocol repeatability and Bland-Altman Limits of Agreement. The conditions were stricter than those previously applied and showed very good repeatability for both systems. Moreover, they highlighted agreement for scapula (1) medio-lateral rotations during humeral forward flexion up to $120^{\circ}$ and during scapular plane abduction up to $100^{\circ}$, and (2) protraction-retraction for humeral flexion and scaption up to $50^{\circ}$. The paper demonstrated the reliability of current measurement methods, but also highlighted areas where we need to improve. The importance of this work in laying the foundations for future multicenter studies led to it being awarded the "ISG Best Methodological Paper Award" by an independent group of reviewers. The award was supported by Springer Verlag, which offers open-access to this paper.

Dynamic factors contributing to rotator cuff disease are further analyzed in the three papers by Henseler et al. [16], de Witte et al. [13] and Marchi et al. [19]. 
Firstly, Henseler and co-workers addressed the problem of early-stage rotator cuff tear diagnosis, based on a novel "active" radiographic technique, suitable for routine clinical use. In particular, they proposed to modify the active abduction view [5], keeping the subject arm alongside the body, but asking the patient to exert an active isometric arm abduction moment. Results confirmed that this technique is capable of highlighting cranial translation in cuff tear patients. Moreover, cranial translation is largest during active abduction and there is significantly more cranial translation in postero-superior cuff tear patients compared to supraspinatus cuff tear patients.

Complementing Henseler's paper, de Witte et al. [13] looked at the outcome evaluation of rotator cuff repair. In particular, they look at deltoid activation pre- and postsurgery with respect to controls, as an easy to measure proxy for rotator cuff functionality. Increased compensatory deltoid activation was found in preoperative rotator cuff tear patients. The postoperative decrease in compensatory deltoid activation, although not significant, could indicate (partially) restored rotator cuff function in at least some patients.

Finally, the paper by Marchi et al. [19] addresses the problem of improving the diagnosis of glenohumeral instability and quantifying the level of impairment, which has been limited so far. The method is highly innovative and combines (1) the controlled application of forces at the hand through a robot during a simple tasks with visual feedback (Haptic Master), (2) model-based estimates of the magnitude and direction of the glenohumeral joint reaction force vector (using the Delft Shoulder and Elbow Model), which provides an estimate of the stability of the joint during a functional task, and (3) EMG recorded from the subject to constrain the muscle activation patterns estimated by the model. Experiments on controls and patients with previously treated glenohumeral instability highlighted significant differences. Glenohumeral stability was lower in the "previously injured" group compared to the controls in all tested directions, even though all participants were back to normal activities and reported no symptoms from their injuries. The method proposed by Marchi and co-workers might prove to be a powerful clinical tool. Moreover, it is a great example of system integration and clinical use of biomechanical models. For all these reasons, it received by an independent group of reviewers the "ISG Best Clinical Paper Award". The award was supported by Springer Verlag, which offers open-access to this paper.

For an in-depth review of current shoulder biomechanical models, the reader of this issue can refer to three papers, i.e., $[3,4,7]$. The first is the review by Bolsterlee, Veeger and Chadwick, which analyzed the application of models as clinical tools, i.e., to answer questions on (1) how the shoulder works from a biomechanical perspective, (2) how different tasks affect joint loading, (3) what happens to shoulder function when the morphology is surgically altered, e.g., after a tendon transfer, and (4) how joint load changes based on inter-individual differences, e.g., subject scaling. This latter is a point of strong debate and constant attention. The authors conclude that "although considerable effort is put into adding complexity to models, for example, by making them subject-specific, we have found little evidence of their superiority over current models. The current trend in development toward individualized, more complex models needs to be justified by demonstrating their ability to answer questions that cannot already be answered by existing models". We are sure this conclusion will stimulate contributions from the scientific community on shoulder biomechanics models.

Bolsterlee and Veeger themselves, with the further contribution of Van der Helm, compare three different approaches to model-to-subject scaling in the paper [4]. The problem they address is that kinematics measured in vivo, when applied on shoulder models (the delft shoulder and elbow model in this case), can violate the closed-chain kinematic constraints. Typically, kinematics is modified to respect these constraints (subject-to-model scaling), but this leads to adjustments in the clavicle and scapula rotations relative to the thorax which can be greater than $8^{\circ}$ (RMSE). Two alternatives are (1) imposing soft constraints, i.e., allowing a limited violation of the constraints, or (2) scaling the model to fit the subject, based on the position of anatomical landmarks. A combination of both decreased the difference between measured and simulated kinematics the most, i.e., by $62 \%$. Most importantly, this was accompanied by only a small deviation from motion constraints $(0.24 \mathrm{~cm}$ on average). Both scaling and using soft constraints independently resulted in an improvement of the match of recordings on simulated shoulder kinematics and can therefore be considered an improvement to the existing method. Interestingly, the effect on the glenohumeral joint contact force was however marginal $(1.3 \%)$. The authors argue that whether new assumptions that are introduced when scaling a model are more valid than the assumptions of a generic model for all subjects, will be matter of debate.

Finally, the paper by Brookham et al. [7] addressed a further long-standing issue in biomechanical models, i.e., the missing or underestimation of muscle co-contraction. In particular, the authors, through an in-depth experimental approach, tried to quantify empirical co-activation ratio. Results were promising for internal rotation exertions, but mixed for external rotations, with no or marginal contribution of PCSA on the co-activation index.

Two further methodological contributions complete the issue $[1,20]$. The former, by Oliver, looks at the relationship between gluteal muscle and upper limb kinematics and kinetics in softball position players, through EMG, kinematics and kinetics measures. This study is among the few 
on softball and the first about mechanics of softball position players. Moreover, it follows a valuable approach, i.e., analyzes kinetic chains rather than single muscles of the upper limb, trunk and lower limbs. Pearson's correlation revealed significant correlation between non-throwing gluteus maximus and elbow moments as well as between trunk flexion/rotation and shoulder moments. Results suggest that the kinematic actions of the pelvis and trunk are strongly related to the actions of the shoulder during throwing.

Finally, the paper by Baumgartner et al. [1] addresses the relevant problem of testing shoulder prostheses in vitro, in conditions close to physiology. For this purpose, the authors designed and tested, with promising results, a shoulder simulator able to perform repetitive, reproducible humeral movements to assess primary stability in testing of prosthesis designs. To achieve predefined glenohumeral angles, individual muscle forces are controlled using realtime, closed-loop control.

\section{Suggestions for future research}

As pointed out by Braman et al. [6], epidemiological literature clearly identifies greater risks for shoulder pathologies in certain occupations and overhead athletes. The papers presented in this issue, dealing with impingement, rotator cuff tears, glenohumeral instability, shoulder prostheses, and muscle synergies, as well as enhanced measurement and estimation techniques by biomechanical models, directly impact issues within these two groups of subjects. In particular, occupational diseases are reaching significant figures, which deserve specific interventions, with the contribution of both researchers and policy makers. For instance, the statistics available from the Italian Workers Compensation Authority (INAIL) point out the high incidence of upper limb and shoulder pathologies. If we consider the work-related accidents which took place in 2012 and were compensated up to April 2013, those related to the upper limb were $38 \%$ of the total, namely 163,850 cases out of 431,437 . Of these, $15 \%$ can be referred to the shoulder, i.e., 24,423 cases. The shoulder is, therefore, the second most affected anatomical region after the hand in the upper extremity (Table 1), and the fifth overall, considering the 20 anatomical regions referred to in the INAIL statistics. Most importantly, injuries at the shoulder are first in terms of average time of "temporary total disability to work", with 37 days compared to an average of 26. Finally, economic compensation rated fifth

Table 1 Italian National statistics provided by INAIL (Italian Workers' Compensation Authority), regarding work-related injuries during 2012, compensated up to April 2013, with specific reference to the shoulder, divided by INAIL-managed sectors

\begin{tabular}{|c|c|c|c|c|}
\hline & Industry and services & Agriculture & State delegation & Total \\
\hline \multicolumn{5}{|l|}{ Shoulder: type of lesion } \\
\hline Strain lesions & 625 & 50 & 4 & 679 \\
\hline External objects & 3 & 1 & & 4 \\
\hline Lesions due to other agents & 58 & 6 & 1 & 65 \\
\hline Lesions due to infective agents and parasites & 3 & & & 3 \\
\hline Anatomical loss & 1 & & & 1 \\
\hline Fracture & 1.980 & 209 & 125 & 2.314 \\
\hline Dislocation, distortion, distraction & 8.584 & 968 & 265 & 9.817 \\
\hline Contusion & 9.469 & 1.305 & 641 & 11.415 \\
\hline Wound & 108 & 16 & 1 & 125 \\
\hline \multicolumn{5}{|l|}{ N.A. } \\
\hline Total at shoulder & 20.831 & 2.555 & 1.037 & 24.423 \\
\hline \multicolumn{5}{|l|}{ Upper limb: all segments } \\
\hline Hand & 88.150 & 7.154 & 1.830 & 97.134 \\
\hline Wrist & 11.381 & 1.562 & 1.022 & 13.965 \\
\hline Forearm/Arm & 17.642 & 1.132 & 407 & 19.181 \\
\hline Elbow & 8.032 & 621 & 494 & 9,147 \\
\hline Shoulder & 20.831 & 2.555 & 1.037 & 24.423 \\
\hline Total number of lesions at upper limb & 146.036 & 13.024 & 4.790 & 163.850 \\
\hline Total number of work-related injuries & 380.042 & 33.678 & 17.717 & 431.437 \\
\hline
\end{tabular}

"National delegation" [ITA: Gestione per conto dello Stato] incorporates a subset of employees of public administrations that are managed by INAIL. Moreover, it incorporates medical doctors exposed to X-rays and radioactive materials that work under public regulation. Source http://bancadaticsa.inail.it/ 
Table 2 Duration of "temporary total disability to work", due to injuries related to the shoulder with indication of the associated average compensation, compared to the same data for the average of the other body regions

Data refer to 2012 injuries compensated up to April 2013. Source http://bancadaticsa.inail.it/

\begin{tabular}{llll}
\hline & Industry and services & Agriculture & Total \\
\hline $\begin{array}{l}\text { Shoulder } \\
\begin{array}{l}\text { Average duration of temporary total disability to } \\
\quad \text { work (days) }\end{array}\end{array}$ & 33.3 & 41.6 & 37.45 \\
$\begin{array}{l}\text { Time rating over body segments } \\
\text { Average compensation }(€)\end{array}$ & 1 & 1 & \\
$\begin{array}{l}\text { Compensation raging } \text { over body segments } \\
\text { Total number of work-related accidents }\end{array}$ & 5 & 1.817 & 2.004 \\
$\begin{array}{l}\text { Average duration of temporary total disability to } \\
\quad \text { work (days) }\end{array}$ & 23.8 & 4 & \\
Average compensation $(€)$ & 1.447 & 29 & 26.4 \\
\hline
\end{tabular}

in the industrial and fourth in the agriculture sectors, with an average of $€ 2,004$ compared to an average of $€ 1,366$ for the other work-related injuries (Table 2).

The situation is even more severe if we take into consideration occupational diseases. By examining the diseases that occurred in 2012 and were compensated up to April 2013, those related to a "biomechanical overload" of the upper limb were $37 \%$ of all compensated occupational diseases $(4,821$ out of 12,920$)$. Just considering the "industrial and services" sector (which is the most complete in terms of data), occupational diseases at the shoulder represented $16 \%$ (1,648 cases) of all occupational diseases (10,047 cases), i.e., $46 \%$ of those related to the upper limb (3,583 cases).

Based on these data, we would encourage further research on (1) quantitative analysis of shoulder loading during tasks associated with musculoskeletal injuries, and ways to reduce that loading, (2) simple and effective tools to improve the diagnosis and outcome assessment of motion-related shoulder diseases and (3) the development of rehabilitation treatments focused on occupational tasks, taking advantage of state-of-the-art biofeedback technologies, and exploiting the power of biomechanical models for muscle force prediction.

\section{Conclusive remarks}

We hope that the studies presented in this issue will stimulate interest in shoulder biomechanics of researchers with a multidisciplinary background. So we invite young and experienced investigators to celebrate with us the upcoming 10th conference of ISG that will be held by the University of Waterloo, Ontario, Canada, from July 13 to 15, 2014.

We would like to thank all the reviewers that helped us with the preparation of this issue as well as the other Board Members of the ISG, John Borstad, Cathy Holt and Andrew Karduna. Finally, we would like to thank Prof. Jos Spaan, former editor-in-chief of this journal, who gave us the opportunity to start (in 2009) and continue the collaboration between ISG and Medical and Biological Engineering and Computing.

Acknowledgments We would like to thank all the people that contributed to the organization of the 9th Conference of the ISG that took place in Aberystwyth, Wales in 2012.

\section{References}

1. Baumgartner D, Tomas D, Gossweiler L, Siegl W, Osterhoff G, Heinlein B (2013) Towards the development of a novel experimental shoulder simulator with rotating scapula and individually controlled muscle forces simulating the rotator cuff. Med Biol Eng Comput Oct 30. [Epub ahead of print] PubMed PMID: 24170552

2. Benedetti MG, Merlo A, Leardini A (2013) Inter-laboratory consistency of gait analysis measurements. Gait Posture 38(4):934-939

3. Bolsterlee B, Veeger DH, Chadwick EK (2013) Clinical applications of musculoskeletal modelling for the shoulder and upper limb. Med Biol Eng Comput 51(9):953-963

4. Bolsterlee B, Veeger HE, van der Helm FC (2013) Modelling clavicular and scapular kinematics: from measurement to simulation. Med Biol Eng Comput Mar 30. [Epub ahead of print] PubMed PMID: 23543279

5. Bloom RA (1991) The active abduction view: a new maneuver in the diagnosis of rotator cuff tears. Skeletal Radiol 20(4):255-258

6. Braman JP, Zhao KD, Lawrence RL, Harrison AK, Ludewig PM (2013) Shoulder impingement revisited: evolution of diagnostic understanding in orthopedic surgery and physical therapy. Med Biol Eng Comput. Apr 10. [Epub ahead of print] PubMed PMID: 23572144

7. Brookham RL, Dickerson CR (2013) Empirical quantification of internal and external rotation muscular co-activation ratios in healthy shoulders. Med Biol Eng Comput June 14. [Epub ahead of print] PubMed PMID: 23765130

8. Cutti AG, Veeger HE (2009) Shoulder biomechanics: today's consensus and tomorrow's perspectives. Med Biol Eng Comput 47(5):463-466

9. Cutti AG, Ferrari A, Garofalo P, Raggi M, Cappello A, Ferrari A (2010) 'Outwalk': a protocol for clinical gait analysis based on inertial and magnetic sensors. Med Biol Eng Comput 48(1):17-25

10. Cutti AG, Giovanardi A, Rocchi L, Davalli A, Sacchetti R (2010) Ambulatory measurement of shoulder and elbow kinematics through inertial and magnetic sensors. Med Biol Eng Comput 46(2):169-178 
11. de Vries WH, Veeger HE, Cutti AG, Baten C, van der Helm FC (2010) Functionally interpretable local coordinate systems for the upper extremity using inertial and magnetic measurement systems. J Biomech 43(10):1983-1988

12. de Witte PB, de Groot JH, van Zwet EW, Ludewig PM, Nagels J, Nelissen RG, Braman JP (2013) Communication breakdown: clinicians disagree on subacromial impingement. Med Biol Eng Comput Apr 25. [Epub ahead of print] PubMed PMID: 23615729

13. de Witte PB, van der Zwaal P, van Arkel ER, Nelissen RG, de Groot JH (2013) Pathologic deltoid activation in rotator cuff tear patients: normalization after cuff repair? Med Biol Eng Comput. July 6. [Epub ahead of print] PubMed PMID: 23832323

14. Ferrari A, Benedetti MG, Pavan E, Frigo C, Bettinelli D, Rabuffetti M, Crenna P, Leardini A (2008) Quantitative comparison of five current protocols in gait analysis. Gait Posture 28(2):207-216

15. Ferrari A, Cutti AG, Garofalo P, Raggi M, Heijboer M, Cappello A, Davalli A (2010) First in vivo assessment of "Outwalk": a novel protocol for clinical gait analysis based on inertial and magnetic sensors. Med Biol Eng Comput 48(1):1-15

16. Henseler JF, de Witte PB, de Groot JH, van Zwet EW, Nelissen RG, Nagels J (2013) Cranial translation of the humeral head on radiographs in rotator cuff tear patients: the modified active abduction view. Med Biol Eng Comput. 2013 Apr 1. [Epub ahead of print] PubMed PMID: 23543305

17. International Shoulder Group, Technical Group of the International Society of Biomechanics. www.internationalshouldergrou p.org

18. Karduna AR, McClure PW, Michener LA, Sennett B (2001) Dynamic measurements of three-dimensional scapular kinematics: a validation study. J Biomech Eng 123(2):184-190

19. Marchi J, Blana D, Chadwick EK (2013) Glenohumeral stability during a hand-positioning task in previously injured shoulders. Med Biol Eng Comput May 24. [Epub ahead of print] PubMed PMID: 23702698

20. Oliver GD (2013) Relationship between gluteal muscle activation and upper extremity kinematics and kinetics in softball position players. Med Biol Eng Comput. [Epub ahead of print] PubMed PMID: 23519517

21. Parel I, Cutti AG, Kraszewski A, Verni G, Hillstrom H, Kontaxis A (2013) Intra-protocol repeatability and inter-protocol agreement for the analysis of scapulo-humeral coordination. Med Biol Eng Comput. Oct 18. [Epub ahead of print] PubMed PMID: 24136689 